\title{
Uso del aditivo mineral como modificador de las propiedades mecánicas en el concreto: una revisión
}

\section{Use of mineral additive as a modifier of the mechanical properties in concrete: a review}

\section{Sócrates Pedro Muñoz Pérez}

Director de la Escuela Profesional de Ingeniería Civil.

Universidad Señor de Sipán. Chiclayo, Perú.

msocrates@crece.uss.edu.pe

\section{Jhonathan Janmarco Burga Carrasco}

Estudiante Escuela Profesional de Ingeniería Civil.

Universidad Señor de Sipán. Chiclayo, Perú.

bcarrascojhonat@crece.uss.edu.pe

\section{Renato Cordova Guerrero}

Estudiante Escuela Profesional de Ingeniería Civil.

Universidad Señor de Sipán. Chiclayo, Perú.

cguerrerorenato@crece.uss.edu.pe

\section{Jesús Alberto Castro Palma}

Estudiante Escuela Profesional de Ingeniería Civil.

Universidad Señor de Sipán. Chiclayo, Perú.

cpalma@crece.uss.edu.pe

\section{Kristell Esteffania Bonilla Bances}

Estudiante Escuela Profesional de Ingeniería Civil. Universidad Señor de Sipán. Chiclayo, Perú.

bbanceskristele@crece.uss.edu.pe

\section{Sandro Enrique Ramos Castillo}

Estudiante Escuela Profesional de Ingeniería Civil. Universidad Señor de Sipán. Chiclayo, Perú.

rcastillondro@crece.uss.edu.pe

Fecha de recepción: 5 marzo 2021 / Fecha de aprobación: 4 octubre 2021

Índices y Bases de Datos:

\section{latindex ucrindex}

\section{- Dialnet DOA}

\section{Ulbiblat ERIHPLUS}

Políticas de Uso:

\section{(c)}

Revista Métodos y Materiales por LanammeUCR se distribuye bajo: Licencia Creative Commons Atribución-NoComercialSinDerivar 4.0 Internacional. ISSN electrónico: 2215-4558 


\section{Uso del aditivo mineral como modificador de las propiedades mecánicas en el concreto: una revisión}

\section{Use of mineral additive as a modifier of the mechanical properties in concrete: a review}

\section{Sócrates Pedro Muñoz Pérez}

Director de la Escuela Profesional de Ingeniería Civil. Universidad Señor de Sipán. Chiclayo, Perú.

msocrates@crece.uss.edu.pe

\author{
Jhonathan Janmarco Burga Carrasco \\ Estudiante Escuela Profesional de Ingeniería Civil. \\ Universidad Señor de Sipán. Chiclayo, Perú. \\ bcarrascojhonat@crece.uss.edu.pe

\section{Renato Cordova Guerrero} \\ Estudiante Escuela Profesional de Ingeniería Civil. \\ Universidad Señor de Sipán. Chiclayo, Perú. \\ cguerrerorenato@crece.uss.edu.pe
}

\author{
Jesús Alberto Castro Palma \\ Estudiante Escuela Profesional de Ingeniería Civil. \\ Universidad Señor de Sipán. Chiclayo, Perú. \\ cpalma@crece.uss.edu.pe
}

\section{Kristell Esteffania Bonilla Bances}

Estudiante Escuela Profesional de Ingeniería Civil. Universidad Señor de Sipán. Chiclayo, Perú.

bbanceskristele@crece.uss.edu.pe

\section{Sandro Enrique Ramos Castillo}

Estudiante Escuela Profesional de Ingeniería Civil. Universidad Señor de Sipán. Chiclayo, Perú.

rcastillondro@crece.uss.edu.pe

Fecha de recepción: 5 marzo 2021 / Fecha de aprobación: 4 octubre 2021

\section{RESUMEN}

El presente artículo recopila e identifica la adición de ciertos aditivos minerales de uso más frecuente en la industria de la construcción como modificadores del concreto durante su estado fresco y endurecido, los cuales en su mayoría son usados como remplazo parcial del cemento. Actualmente los investigadores buscan optimizar las propiedades del diseño de mezclas dependiendo al uso por el cual son concebidas, así mismo que sea factible económicamente y respetuoso con el medio ambiente. Se revisaron 50 artículos indexados entre los años 2010 y 2021 distribuidos en las siguientes bases de datos: 37 artículos de Scopus, 10 artículos de ScienceDirect, 2 artículos de Springer y 1 artículo de SciELO. Los aditivos en estudio son escoria de alto horno granulada (EAHG), cenizas volantes (CV), humo de sílice (HS), ceniza de cascarilla de arroz (CCA). En su mayoría presentan una mejora considerable en sus características mecánicas de tracción, resistencia a la compresión y módulo de elasticidad. La adición de CV mejora en la mezcla del diseño durante su estado fresco, la CCA durante su estado endurecido y las mejores resistencias para el HS; sin embargo, la EAHG resulta cumplir con casi todas las expectativas en mayor porcentaje y así mismo junto con la CCA, son potencialmente competitivos.

PALABRAS CLAVE: Escoria de alto horno, cenizas volantes, humo de sílice, ceniza de cascarilla de arroz, propiedades, aditivo mineral.

\section{ABSTRACT}

This article collects and identifies the addition of certain mineral additives most frequently used in the construction industry as modifiers of concrete during its fresh and hardened state, most of which are used as a partial replacement for cement. Currently researchers seek to optimize the properties of the design of mixtures depending on the use for which they are conceived, as well as that it is economically feasible and respectful with the environment. 50 articles indexed between 2010 and 2021 distributed in the following databases were reviewed: 37 articles from Scopus, 10 articles from ScienceDirect, 2 articles from Springer and 1 article from SCIELO. The additives under study are granulated blast furnace slag (EAHG), fly ash (CV), silica fume (HS), rice husk ash (CCA). Most of them present a considerable improvement in their mechanical characteristics of traction, resistance to compression and modulus of elasticity. The addition of CV improves the mix of the design during its fresh state, the CCA during its hardened state and the best resistances for the HS; However, the EAHG turns out to meet almost all expectations in a higher percentage and likewise, together with the CCA, they are potentially competitive

KEYWORDS: Blast furnace slag, fly ash, silica fume, rice husk ash, properties, mineral additive. 


\section{INTRODUCCIÓN}

A medida que transcurre el tiempo el uso del concreto convencional en las construcciones sigue siendo el componente líder gracias a sus propiedades mecánicas, rendimiento, fácil colocación y rentabilidad. Sin embargo, la forma de su fabricación es un tema preocupante a los ojos de profesionales del sector ambiental, puesto que se ha estimado entre un 5 a $7 \%$ de $\mathrm{CO}_{2}$ que acelera de forma gradual al calentamiento global. A través de la creciente industrialización se ha reciclado tales desechos con el fin de reducir el uso del cemento Portland, utilizando como materiales suplementarios a cenizas volantes $(\mathrm{CV})$, escoria granulada de alto horno (EAGH), etc. (Cao, et al., 2017).

Aproximadamente desde los años 80 se ha usado en la construcción una mejora en el diseño del concreto o lo que se conoce como HPC (concreto de alto rendimiento); que incorpora superplastificantes y aditivos minerales, obteniendo hasta resistencias a la compresión con más de $60 \mathrm{MPa}$ ( $600 \mathrm{~kg} / \mathrm{cm} 2$ ) (Kaïkea, et al., 2014). Existen además otras modificaciones como el concreto autocompactante (SCC) cuyas propiedades le dan la capacidad de compactarse sin requerir el vibrado habitual, segregación y exudación; es decir llena por su propio peso los vacíos que se generan durante su estado fresco (Siad, et al., 2014); puede disminuir en un $20 \%$ el uso del cemento con ayuda de incorporaciones minerales incrementando una mayor durabilidad que el concreto convencional, (Soares dos Anjos, et al., 2020). (Anand, et al., 2016) investigaron cómo reaccionarían las propiedades del SCC a altas temperaturas colocando la fusión mineral de $\mathrm{HS}, \mathrm{CV}$ y metacaolín (MK) a $900^{\circ} \mathrm{C}$; resultando que este último mineral reaccionaría mejor a compresión, tracción, resistencia exural y módulo de elasticidad que los otros dos minerales que protege mejor al concreto del desconchado; sin embargo (Reddy \& Ramaswamy, 2017) aseguran que por sí solo falla a $398^{\circ} \mathrm{C}$ en la edad de 28 días y $575^{\circ} \mathrm{C}$ a los 365 días.

Realizar combinaciones con agregados RAP está siendo una novedosa técnica de incorporar el aditivo mineral con muestras recicladas del pavimento (Singh, et al., 2017) estudiaron a 3 aditivos minerales diferentes $\mathrm{HS}, \mathrm{CV}$ y ceniza de bagazo de caña $(\mathrm{CBC})$, actuando como remplazo del peso de cemento mostrando mejoras con porcentajes de 10, 20 y $5 \%$ respectivamente en sus propiedades mecánicas y exceder el 5\% más en CBC aumenta la absorción de agua en tanto a los vacíos permeables totales. Otros tipos de agregados de concreto reciclados o denominados RCA con adiciones de MK y HS de un $10 \%$ al diseño de mezcla de SCC son capaces de compensar las pérdidas de las propiedades de durabilidad del concreto de manera óptima cuando son reemplazadas parcialmente del agregado natural en un 50\% que cuando se reemplaza en un 100\% (Kapoor, et al., 2016); cabe decir que la utilizacion del RCA no son frecuentes y eso debido a su alta porosidad y capacidad de absorción de agua (Bouarroudj, et al., 2021). Los agregados reciclados de cerámica en combinación con el MK y HS resultan ser factibles en la elaboración del concreto de ultra alto desempeño (UHPC) mejorando la trabajabilidad y resistencias óptimas con un reemplazo de cemento de hasta $20 \%$ de un $133,1 \mathrm{MPa}(\cong 1331 \mathrm{~kg} / \mathrm{cm} 2)$ a un $146,6 \mathrm{MPa}(\cong 1466 \mathrm{~kg} / \mathrm{cm} 2)$ a los 28 días (Amin, et al., 2020).

Para ciertas combinaciones en el diseño puede resultar favorable adherir los aditivos minerales; sin embargo, para otras no es la fusión más recomendable como lo es para el bambú donde (Karthik, et al., 2017) investigaron que el uso de las tiras de esta fibra e incorporación de aditivo de CV y EAHG como sustito del $25 \%$ del cemento y arena manufacturada no presenta la mejor resistencia exural que cuando se adhieren a la mezcla con bambú material convencional.

Esta literatura pretende generar un amplio conocimiento en tanto a la influencia de los aditivos minerales de HS, CV, EAHG, CCA como material suplementario parcial del cemento; además de analizar y comparar las modificaciones en sus propiedades mecánicas y microestructura del concreto puzolánico.

\section{DESARROLLO}

\subsection{MATERIALES CONSTITUYENTES}

La EAHG es un subproducto que se obtiene de la fabricación de hierro durante el proceso de extracción como desecho; compuesto principalmente por magnesio y aluminosilicatos de calcio; este componente mejora la trabajabilidad, durabilidad y a pesar que inicialmente la resistencia disminuye; llega alcanzar una resistencia tardía esperada (Liu, et al., 2014). Este elemeto puede mejorar la resistencia del concreto expuestos a ciclos de congelamiento y deshielo; sin el uso de agentes incorporadores de aire (Toshiki \& Takashi, 2021).

Las CV provienen principalmente de las centrales eléctricas térmicas como un subproducto de desecho de la quema del carbón pulverizado; como alternativa para alcanzar un desarrollo sostenible se utiliza en proporciones de 50 a $60 \%$ de CV y así obtener lo que se conoce como cenizas volantes de alto volumen (HVFA) (Wang \& Park, 2015). La ceniza volcánica metamórfica constituye cerca del $25 \%$ y todas ellas han sido alteradas (Shi, et al., 2020). Son ricas en alúmina y sílice; cuando se activan produce un gel de aluminosilicato que actúa como aglutinante. 
La CCA son obtenidas de la industria de molienda después de la producción y cosechas de arroz; al quemarse genera una ceniza oscura y es utilizada como combustible en la generación de electricidad; este residuo es vertido en grandes áreas y no se elimina convenientemente (Rodrigues \& Beraldo, 2010). Contiene en peso de sílice entre un 90 a $95 \%$; los cuales dependen de la temperatura de calcinación y la forma de producción en cenizas (Sathawane, et al., 2013)

El HS es un material altamente puzolánico como subproducto de la industria del ferrosilicio mejora las propiedades mecánicas y de durabilidad en el concreto en tanto a su resistencia y reduce parcialmente la permeabilidad (Nagrockiene, et al., 2019). El componente de sílice en sus propiedades reacciona en el concreto reduciendo el hidróxido de calcio; acortando así la porosidad; tiene una naturaleza única para aumentarla dureza del concreto debido a su alta densidad (Sanjay, et al, 2020).

\subsection{INFLUENCIA DEL ADITIVO EN ESTADO FRESCO}

Existen diferentes métodos de simulación de flujo del concreto durante su estado fresco; es decir en el periodo de mezcla, transporte colocación y compactación.

Cao y colaboradores analizaron los efectos de CV y EAHG sobre la dependencia del tiempo en el concreto fresco utilizando el método de elementos discretos (DEM) donde realizan diferentes pruebas de flujo en relación al tiempo con un embudo; los resultados demostraron que este método puede predecir la fluidez en función tiempo del concreto y que, en comparación con las $\mathrm{CV}$, la extensión decreciente de la EAHG es mayor (Cao, et al., 2017).

Otro tipo de ensayos en estado fresco son por ejemplo con el VSI (índice de visual) que básicamente mide la estabilidad de mezclas en el concreto; seguido de su fluidez midiendo el diámetro de concreto esparcido y el tiempo T50 (Ahmed \& Najim, 2016), la prueba de embudo (V-embudo) también está adaptándose en pruebas de ensayos en edad fresca; mediante el cual se determinan las propiedades de la muestra dentro de los 20 minutos posteriores a la colocación del agua. Los investigadores Zeyad y Saba incorporaron al SCC adiciones del 20 y $40 \%$ de CV como reemplazante de la masa del cemento resultando positivamente aumentando así la exudación y segregación; en cambio adiciones de un $60 \%$ resulta perjudicial; sin embargo, el proceso del embudo fue dificultoso; exhortando llevar a cabo conforme a las especificaciones correspondientes (Saba \& Zeyad, 2018).

Boukendakdji y colaboradores estudiaron la incorporación de la EAHG en porcentajes de 10, 15, 20 y $25 \%$ en reemplazo de masa de cemento; además de realizar pruebas de asentamiento V-Funnel, J-Ring, U-Box, siguiendo el método de Okamura con mejoras; que en principio fija el intervalo de $2-7 \%$ de contenido de aire; de los áridos gruesos en $50 \%$ y el mortero debe contener $40 \%$ de árido fino, seguidamente la cantidad de superplastificante se mide a través de ensayos, obteniendo así que dicha sustitución es beneficiosa para el concreto fresco mejore en un $20 \%$ su trabajabilidad (Boukendakdji, et al., 2012).

La adaptación de uno de los métodos más conocido para ensayos del concreto fresco lo usó Das y colaboradores con el ensayo de cono de asentamiento donde adiciona a su diseño desde 0 a $20 \%$ en peso de CCA y observo que al aumentar al máximo el porcentaje de aditivo mineral, disminuye el flujo del concreto fresco en su trabajabilidad (Das Bheel, et al., 2019).

En la investigación de Hariharan y colaboradores determinaron la prueba de asentamiento en los minerales de $\mathrm{HS}$ y $\mathrm{CV}$ en el concreto fresco alcanzando un máximo de $165 \mathrm{~mm}$; con la adición de 6 y 10\% de reemplazo del cemento; mientras aumenta el porcentaje de HS disminuye el asentamiento; una respuesta contraria es con la adición de CV de 30, 40 y 50\% que al aumentar reduce la viscosidad plástica destacando una mejor trabajabilidad (Hariharan, et al. , 2011); además se sabe que los aditivos que mejoran la viscosidad afectan la calidad del concreto en el proceso de desencofrado de desecho (Lin \& Huang, 2015) y en el caso que se lleven a cabo un curado acelerado provoca una tasa de hidratación y resultan formaciones de microestructura prematuras (Sukontasukkul, et al., 2020)

En la tabla 1 se describe un resumen de las propiedades del concreto en estado freso, afectado por el aditivo mineral; detallando los pesos unitarios de material cementoso, del aditivo, del agregado fino y grueso. 


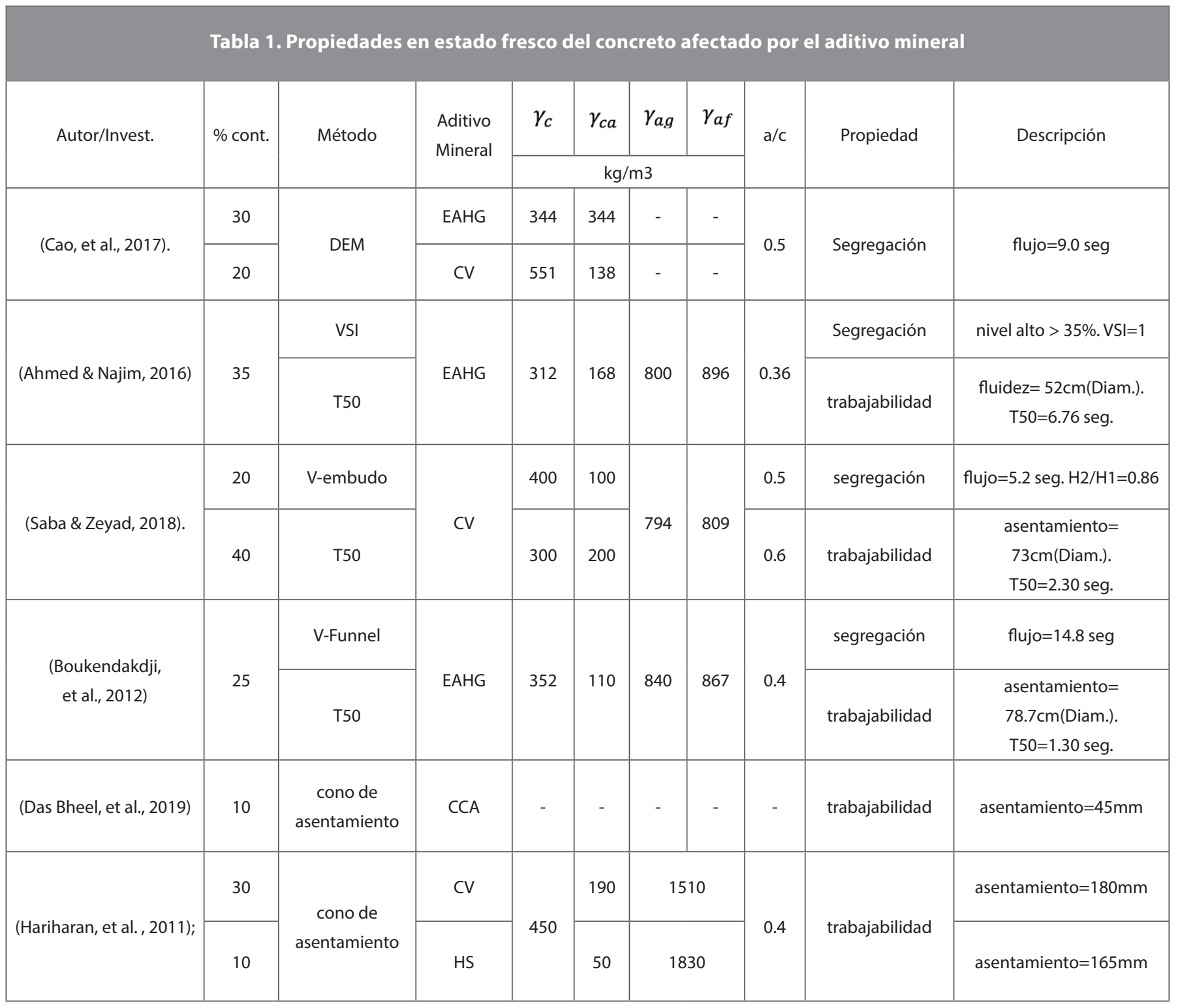

\subsection{INFLUENCIA DEL ADITIVO EN ESTADO ENDURECIDO}

El proceso de endurecimiento se ve afectado de cierta manera según el espécimen de probeta que se crea mayormente en concretos de alta resistencia y rendimiento. $\mathrm{Hu}$ y sus colaboradores midieron la contracción autógena y calor de hidratación mediante el método de diseño factorial a partir del conteo de huecos de aire por relaxometría H MNR; en comparación de resultados de los minerales las $\mathrm{CV}$ resultan ser más vulnerables que la EAHG en respuesta a la contracción autógena cuando se disminuye gradualmente la relación a/c y contrariamente cuando se aumenta; el calor de hidratación también (Hu, et al., 2017).
En concordancia con anteriores aportes de profesionales; cada tipo de aditivo dispone de propiedades peculiares para cada uso correspondiente del concreto; por ejemplo, diseñar una cerámica porosa según Ali y colaboradores, es recomendable utilizar la CCA como fuente de sílice y la alúmina como agente formador de poros; utilizar adiciones de 10 a 50\% resulta hasta un 49,04\% más de porosidad y en tanto a dureza 150,92 HV y 158,93 HV (Ali, et al., 2017). Las propiedades de endurecimiento optimas se determinan con un 5\% de CCA y 5\% de CV en reemplazo del cemento (Bheel, et al., 2020). Otro tipo de diseño en relación a altas temperaturas es fabricar un prototipo de concreto laterizado, que viene a ser un concreto con agregados de laterita rico en hierro, y alúmina a menudo utilizado en protecciones 
contra incendios; que en conjunto con las bondades del aditivo mineral; por experimentación, CV y EAHG soporta temperaturas hasta $400^{\circ} \mathrm{C}$;la incorporación de $20 \%$ para CV y $25 \%$ para EAHG, soporta el doble de temperatura $\left(800^{\circ} \mathrm{C}\right)$ donde desarrolla fisuras térmicas (Mathew \& Paul, 2014).

En necesidad de encontrar un concreto que posea la capacidad de enfrentar los ataques externos patológicos y espontáneoquímico de su microestructura, Deppa y Felix realizan la combinación experimental del HS y EAHG para determinar el comportamiento autocurativo del concreto; induciendo a la mezcla del primer aditivo entre un 2.5 a $12.5 \%$, y como masa reemplazante al cemento el segundo entre 35 y 55\%; demostrando que mientras menor sean las partículas de la composición existirán mayor cantidad de partículas no hidratadas que cuando entren en contacto con la humedad atmosférica la reacción de los aditivos formaran hidróxido de calcio que por medio de las aberturas lograran el efecto de autocuración (Depaa et al., 2015).

\begin{tabular}{|c|c|c|c|c|c|c|c|c|}
\hline Autor/Invest. & $\%$ & Aditivo & $\gamma_{c a}$ & $a / c$ & $\sigma_{c}$ & $\sigma_{t}$ & $\sigma_{f}$ & $E_{c}$ \\
\hline & & & Propor. & & \multicolumn{4}{|c|}{ (28 días) $\mathrm{MPa}$} \\
\hline (Damayanti, et al., 2020) & 10 & CCA & 51.25 & 0.4 & 35.86 & 3.89 & - & - \\
\hline \multirow{2}{*}{ (Bheel, et al., 2020) } & \multirow{2}{*}{5} & $\mathrm{CV}$ & \multirow{2}{*}{ 01:02:04 } & \multirow{2}{*}{0.55} & 32 & 3.6 & - & - \\
\hline & & CCA & & & 25 & 2.8 & - & - \\
\hline \multirow{2}{*}{ (Mathew \& Paul, 2014) } & 25 & EAHG & 93.75 & \multirow{2}{*}{0.42} & 27.92 & 3.24 & 4.48 & 29358 \\
\hline & 20 & $\mathrm{CV}$ & 75 & & 29.73 & 2.9 & 4.29 & 28200 \\
\hline (Pereira, et al, 2015) & 5 & CCA & 25.5 & 0.4 & 60.4 & 5.8 & & 41000 \\
\hline (Attah, et al., 2020) & 40 & EAHG & 171.44 & 0.4 & 58 & 7 & 9 & 35144.3 \\
\hline (Kishor, et al., 2020). & 10 & CCA & 39.1 & 0.45 & 55 & 4.5 & 7.5 & - \\
\hline (Ayesha, et al., 2018) & 10 & CCA & 46.13 & 0.4 & 35 & 9.75 & 16.56 & - \\
\hline (Ahmed, et al, 2020) & 10 & CCA & 45 & 0.4 & 60 & 4.6 & 4.6 & - \\
\hline \multirow{3}{*}{$\begin{array}{c}\text { (Mohamed \& Najm, 2017; } \\
\text { Mohamed \& Al Hawat, 2018) }\end{array}$} & 15 & HS & 72 & \multirow{3}{*}{0.36} & 95.3 & - & - & - \\
\hline & 20 & $\mathrm{CV}$ & 96 & & 67.8 & - & - & - \\
\hline & 35 & EAHG & 168 & & 81 & - & - & - \\
\hline
\end{tabular}




\subsection{CARACTERÍSTICAS MECÁNICAS DE RESISTENCIA COMPRESIVA, TRACTIVA Y MÓDULO DE ELASTICIDAD}

Cierta característica es la principal prioridad que la investigación debe determinar puesto que todo concreto estructural; sin excepción, está diseñado para soportar cargas. (Pereira, et al, 2015) determinaron en una primera fase las características físico-químicas y mecánicas del concreto con CCA en un remplazo del 5 y $10 \%$ de peso cementante mediante un curado térmico y húmedo; para el primer porcentaje resulto ser la compresión más óptima alcanzando un 60,4 MPa a los 28 días y en una segunda fase la tracción represento el $10 \%$ de la compresión; sin embargo, para obtener un concreto de alta resistencia SCC según Ahmed y colaboradores resultaron opuestos para un 10\%, $60 \mathrm{MPa}$ y $5 \%$, $57 \mathrm{MPa}(450 \mathrm{~kg} / \mathrm{m} 3$ de contenido cementoso) y en tracción un porcentaje del 7,6\% de compresión (Ahmed, et al, 2020). Las variedades de combinaciones se llevan a cabo con el fin de buscar las mejores propiedades al concreto tanto así que se probaron el uso de fibras y aditivo mineral, probándose la espina de pescado y CCA con 0,5 y $1 \%$ en fibra y $10 \%$ en aditivo observándose que ocurre una resistencia optima tardía de 56 días en comparación al concreto convencional, con la explicación que la tasa de hidratación de las partículas de cenizas descompuestas por encima de $45 \mu \mathrm{m}$ retiene el aumento de resistencia del concreto; sin embargo a tracción es menor que la del concreto normal (Damayanti, et al., 2020). Así mismo también se ha hecho común sustituir los agregados como la concha triturada; pero se debe tener en cuenta que con un $10 \%$ puede alcanzar resultados óptimos en tanto a resistencias y $20 \%$ de CCA para sustituir al cemento; se emplearía la misma opción de porcentajes para mezclar con EAHG (Kishor, et al., 2020).

Se debe tomar en cuenta la proporción de CCA y otras adiciones para que no afecte el rango de resistencia prevista en su diseño; pues se supone experimentalmente que $15 \%$ reduce gradualmente la fuerza compresiva, y que por tanto se recomienda no superar el 10 a 12\%; al igual que la tracción en su relación a/c que mientras esta sea mayor, la porosidad también ascenderá, en este caso el porcentaje más adecuado es hasta un 10\% (Ayesha, et al., 2018); con lo que respecta a los costos del cemento se reduce en un 31,5\% de sustitución parcial (Hu, et al., 2020). Por otro lado cuando se busca diseñar un tipo de concreto permeable para pavimentos es recomendable el HS que logra alcanzar 52,4 $\mathrm{MPa}$ con un $9 \%$ de masa reemplazante en resistencia pero para una permeabilidad optima se recomienda $6 \%$ que resulta un $208 \%$ más permeable y $16 \%$ menos resistente (Huang, et al., 2020)
El concreto SCC opta por diferentes aditivos minerales para una mejora en sus propiedades mecánicas por lo que Mohamed y Najm mediante un programa experimental multivariable buscando hacer sostenible el uso; en reemplazo del cemento, de CV, HS y EAHG; adicionando además un aditivo reductor de agua de alto rango (HRWA) a base de éter policarboxílico y se mantuvo constante en un 1,5\% de masa de materiales cementosos, por cada adición independiente obtuvieron: primero para las CV con 10, 30 y $40 \%$ una resistencia de $67,96 \mathrm{MPa}$, para el $\mathrm{HS}$ se adiciono un $15 \%$ resultando una fuerza compresiva de 95,3 MPa que representa un 44,2\% más alto que la del patrón; por último la EAHG donde se encontró la relación optima en un 35\% hasta $81 \mathrm{MPa}$; todo ello bajo la edad de 28 días (Mohamed \& Najm, 2017), otra de las investigaciones donde utilizaron las mismas variables y HRWA; en este caso analizar las propiedades mecánicas bajo la denominación de compuestos cementosos de ingeniería (ECC); sobre sistemas binarios y ternarios demostrando que para la primera, la resistencia compresiva disminuye cuando aumenta el contenido en un solo mineral, en especial las $\mathrm{CV}$, para las ternarias las muestras de ECC la compresión es proporcional a medida que se prolonga la edad de curado (Zhu, et al., 2014). (Santhosh \& Arivalagan, 2018) usaron como agregado un tipo de arena manufacturada; la mezcla del diseño contiene un 60\% de EAHG, 30\% de CV y $10 \%$ de HS como reemplazo en conjunto del cemento; lo cual en la experiencia " $X_{4}$ " representó el $60 \%$ de mezcla y $40 \%$ de cemento; siendo el más óptimo en resistencias alcanzando a compresión $24,8 \mathrm{~N} / \mathrm{mm} 2((\cong 240,8 \mathrm{~kg} / \mathrm{cm} 2)$ y en tracción un $9 \%$ del anterior cumpliendo los estándares de un concreto estructural; así mismo representa la alternativa más rentable de reemplazar el cemento en cierto porcentaje.

Uno de los estudios a la resistencia compresiva de un concreto SCC con incorporaciones de CV lo hicieron Mohamed y Al Hawat además de investigar la resistencia a la penetración de cloruros; observaron que lo óptimo que puede alcanzar la adición del 20\% de CV es de 67,96 MPa de compresión y de penetración de cloruros en edad temprana de 14 días con el mismo porcentaje (Mohamed \& Al Hawat, 2018). Los residuos de construcción y demolición son desafortunadamente inaceptables para un concreto estructural; precisamente por su microestructura porosa (Cuenca-Moyano, et al., 2014); pero cuando la combinación adhiere HCF (mezcla de cenizas con alto contenido de calcio) a un LCF (mezcla de cenizas con bajo contenido de calcio) y agregado reciclado; la sustitución del 50\% aumenta significativamente la resistencia a la compresión y disminuye la porosidad; además de ello ofrece resistencia a los ácidos sulfúricos; llegando a un 46,6 $\mathrm{MPa}$ el más óptimo (Nuaklong, et al., 2019). 
Temiz y Kantarci en la obtención de un nuevo material aglutinante mezclaron polvo de calcita, piedra caliza, y CV; sin embargo, no contemplaron los resultados poco optimistas en tanto a resistencias en los dos primeros; a pesar que se obtuvieron a edad temprana altas magnitudes; en edades posteriores ocurrió lo contrario, pero salvó la adición de CV en porcentajes del 30\% (Temiz \& Kantarci, 2014); la misma proporción del mineral con diferentes materiales cementantes entre CV y fibra de ceniza de cascara de coco demuestran; además de una buena resistencia que igualan al del cemento normal (66,2 MPa); pueden poseer la capacidad de convertir el hidróxido de calcio de la misma pasta del cemento en silicato de calcio hidratado (Bayuaji, et al., 2016).

(Ahmed \& Najim, 2016), observaron mediante ensayos netos a tracción con minerales de EAHG que reemplazar un $45 \%$ de masa cementante puede aumentar de manera proporcional la resistencia a compresión para diferentes tipos de muestras; además de realizar un análisis de regresión por medio del denominado IAE (error absoluto integral) para los diferentes códigos de diseño donde demuestran que subestiman las fórmulas de predicción por resistencia a tracción.

Para la obtención del módulo de Young; además de los resultados teóricos es necesario la aproximación más exacta cuando se lleva a cabo de manera experimental; sobre todo en la determinación de deflexiones, cuando se prueban a flexión, por ejemplo, vigas de sección longitudinal y en L; además de ello se trate del tipo de tecnología de geopolímeros con adición del $40 \%$ de EAHG en comparación con la del concreto convencional (CC) la relación de tensión versus deformación aumenta en un 34 y $20 \%$; los resultados experimentales se miden en un software (ANSYS) y al compararse se observan magnitudes más conservadoras (Antonyamaladhas, et al., 2016). La teoría de Schaeffer para el diseño de celosía simplex también está siendo usado como alternativa en la obtención de la optimización de resistencia a la compresión mediante la formulación de un polinomio de segundo grado (Attah, et al., 2020).

El comportamiento mecánico del HS muestra óptimos resultados a compresión desde edades tempranas; así mismo en la adición gradual a la mezcla compuesta es predominante y a una edad tardía de 90 días la tracción es mayor a la del concreto (Liu \& Wang, 2017).

En la tabla 2 se muestra un resumen de las propiedades endurecidas y mecánicas del concreto afectado por el aditivo mineral.

\section{CONCLUSIONES}

El objetivo principal de la presente revisión es de presentar a los investigadores una idea clara de lo que significa trabajar con este tipo de aditivos minerales; sobre todo que aspectos se debe tener en cuenta; antes y después de las experimentaciones resaltando los aportes positivos y negativos de sus propiedades mecánicas; se resume en las siguientes conclusiones:

- Las CV y EAHG tienen las mejores características durante su estado fresco con porcentajes entre el 40 y $20 \%$ respectivamente, aumentando la exudación, segregación y una mejor trabajabilidad.

- Las CCA como agente formador de poros resulta ser la mejor alternativa con adiciones del 10\%; aumentando hasta un 49,04\%; mientras que la EAHG actúa mejor en respuesta a la contracción autógena con la disminución gradual de la relación a/c.

- La resistencia compresiva más óptima sucede cuando se adiciona HS; como reemplazante parcial del cemento; con un 10 y $15 \%$ resultando 95,3 $\mathrm{MPa}$; el segundo más óptimo; la EAHG con un 35\% alcanzando $81 \mathrm{MPa}$; las $\mathrm{CV}$ con un $20 \%$ resistencias hasta de $67,96 \mathrm{MPa}$; y por ultimo las CCA con un 5\% 60,4 MPa, además de ello proporciona el mejor comportamiento a tracción con un $10 \%$ de compresión; por supuesto tratándose de concreto SCC medidos a los 28 días.

\section{REFERENCIAS}

Ahmed, A., Hyndman, F., Kamau, J., \& Fitriani, H. (2020). Rice husk ash as a cement replacement in high strength sustainable concrete. Materials Science Forum, 1007, 90-98. doi:10.4028/ www.scientific.net/MSF.1007.90

Ahmed, O., \& Najim, O. (2016). Splitting Tensile Strength of SelfConsolidating Concrete Containing Slag 1. Conference: Toronoto'2016 AES-ATEMA 29th International ConferenceAt: Toronto, Canada, (pp. 110-116). Toronto.

Ali, M., Hanim, M., Tahir, S., Jaafar, C., Mazlán, N., \& Amin Matori, K. (2017). The Effect of Commercial Rice Husk Ash Additives on the Porosity, Mechanical Properties, and Microstructure of Alumina Ceramics. Advances in Materials Science and Engineering, 2017, 2586026. doi:10.1155 / 2017/2586026

Amin, M., Tayeh, B., \& Agwa, E. (2020). Effect of using mineral admixtures and ceramic wastes as coarse aggregates on properties of ultrahigh-performance concrete. Journal of Cleaner Production, 273. doi:10.1016 / j.jclepro.2020.123073 
Anand, N., Antony Godwin, I., \& Prince Arulraj, G. (2016). Influence of mineral admixtures on mechanical properties of selfcompacting concrete under elevated temperature. Fire and Materials, 40(7), 940-958. doi:10.1002/fam.2353

Antonyamaladhas, Chachithanantham, S., \& Ramaswamy, A. (2016). Performance and Behaviour of Ground Granulated Blast Furnace Slag Imparted to Geopolymer Concrete Structural Elements and Analyzed with ANSYS. Advances in Materials Science and Engineering, 2016(2), 1-9. doi:10.1155/2016/7023897

Attah, I., Kufre Etim, R., Uwadiegwu Alaneme, G., \& Bassey Bassey, O. (2020). Optimization of mechanical properties of rice husk ash concrete using Scheffe's theory. SN Applied Sciences, 2(5), 1-13. doi:10.1007/s42452-020-2727-y

Ayesha, Abdullah, M., Hedayet Ali, M., \& Hedayet Ali, M. (2018). Study on concrete with rice husk ash. Innovative Infrastructure Solutions, 3(1), 1-8. doi:10.1007/s41062-018-0127-6

Bayuaji, R., Kurniawan, R., Yasin, A., Fatoni, H., \& Lutfi, F. (2016). The effect of fly ash and coconut fibre ash as cement replacement materials on cement paste strength. IOP Conference Series: Materials Science and Engineering, 128, p. 012014. Indonesia. doi:10.1088/1757-899X/128/1/012014

Bheel, N., Ali Jokhio, M., Ahmed Abbasi, J., Bux Lashari, H., Imran Qureshi, M., \& Salam Qureshi, A. (2020). Rice Husk Ash and Fly Ash Effects on the Mechanical Properties of Concrete. Engineering, Technology and Applied Science Research, 10(2), 5402-5405. doi:10.48084/etasr.3363

Bouarroudj, M., Rémond, S., Bulteel, D., Potier, G., Michel, F., Zhao, Z., \& Courard, L. (2021). Use of grinded hardened cement pastes as mineral addition for mortars. Journal of Building Engineering, 34. doi:10.1016 / j.jobe.2020.101863

Boukendakdji, O., Kadri, E.-H., \& Kenai, S. (2012). Effects of granulated blast furnace slag and superplasticizer type on the fresh properties and compressive strength of self-compacting concrete. Cement and Concrete Composites, 34(4), 583-590. doi: 10.1016/j.cemconcomp.2011.08.013

Cao, G., Li, Z., \& Guo, K. (2017). Analytical study on the change of fluidity of fresh concrete containing mineral admixture with rest time. Journal of Advanced Concrete Technology, 15(11), 713-723. doi:10.3151/jact.15.713

Cuenca-Moyano, G., Martín-Morales, M., Valverde-Palacios, I., Valverde-Espinosa, I., \& Zamorano, M. (2014). Influence of presoaked recycled fine aggregate on the properties of masonry mortar. Construction and Building Materials, 70, 71-79. doi: 10.1016/j.conbuildmat.2014.07.098

Damayanti, S., Aulia, T., \& Hayati, Y. (2020). The effect of fishbone fiber and rice husk ash additive on the mechanical properties of normal concrete. IOP Conference Series: Materials Science and Engineering, 933, p. 012036. Indonesia. doi:10.1088/1757899X/933/1/012036
Das Bheel, N., Wahab Abro, A., Ali Shar, I., \& Hussain Shaikh, Z. (2019). Use of Rice Husk Ash as Cementitious Material in Concrete. Engineering, Technology and Applied Science Research, 9(3), 4209-4212. doi:10.1007/s41062-018-0127-6

Depaa, R., \& Felix Kala, T. (2015). Experimental investigation of self healing behavior of concrete using Silica fume and GGBFS as mineral admixtures. Indian Journal of Science and Technology, 8(36), 87644. doi:10.17485/ijst/2015/v8i36/87644

Hariharan, A., Santhi , A., \& Mohan , G. (2011). Study on Strength Development of High Strength Concrete Containing Fly ash and Silica fume. International Journal of Engineering Science and Technology, 3(4), 2955-2961.

Hu, L., He, Z., \& Zhang, S. (2020). Sustainable use of rice husk ash in cement-based materials: Environmental evaluation and performance improvement. Journal of Cleaner Production, 264, 121744. doi:10.1016/j.jclepro.2020.121744

Hu, X., Shi, C., Shi, Z., \& Tong, B. (2017). Early age shrinkage and heat of hydration of cement-fly ash-slag ternary blends. Construction and Building Materials, 153, 857-865. doi:10.1016/j. jksues.2016.12.003

Huang, J., Luo, Z., \& Khan, M. (2020). Impact of aggregate type and size and mineral admixtures on the properties of pervious concrete: An experimental investigation. Construction and Building Materials, 265, 120759. doi:10.1016 / j.conbuildmat.2020.120759

Kaïkea, A., Achoura, D., Duplan, F., \& Rizzuti, L. (2014). Effect of mineral admixtures and steel fiber volume contents on the behavior of high performance fiber reinforced concrete. Materials and Design, 63, 493-499. doi:10.1016/j. matdes.2014.06.066

Kapoor, K., Singh, S., \& Singh, B. (2016). Durability of self-compacting concrete made with Recycled Concrete Aggregates and mineral admixtures. Construction and Building Materials, 128, 67-76. doi:10.1016/j.conbuildmat.2016.10.026

Karthik, S., Rao, P., \& Awoyera, P. (2017). Strength properties of bamboo and steel reinforced concrete containing manufactured sand and mineral admixtures. Journal of King Saud University - Engineering Sciences, 29(4), 400-406. doi:10.1016/j. jksues.2016.12.003

Kishor , P., Bhusan Gouda, B., \& Mohapasayat, P. (2020). Effect of Ground Granulated Blast Furnace Slag on the Properties of Sea Shell Concrete. Series: Materials Science and Engineering, Volume 970, Online National Conference on "SUSTAINABLE MATERIAL AND PRACTICES FOR INFRASTRLCTURE DEVELOPMENT" (SMPID-2020) 17th-18th September 2020, Odisha, India, 970, p. 012018. doi:10.1088 / 1757-899X / 970/1/012018 
Kishor, C., Subhrasweta, B., \& Shradha, J. (2020). Effect of rice husk ash on mechanical properties of concrete containing crushed seashell as fine aggregate. Materials Today: Proceedings, 32, 1-6. doi:10.1016/j.matpr.2020.04.049

Lin, C.-Y., \& Huang, W.-T. (2015). Effect of mineral viscosityenhancing admixtures on the solidification of evaporator concentrates. Journal of Hazardous Materials, 298, 294-302. doi:10.1016/j.jhazmat.2015.05.048

Liu, J., \& Wang, D. (2017). Influence of steel slag-silica fume composite mineral admixture on the properties of concrete. Powder Technology, 320, 230-238. doi:10.1016/j.powtec.2017.07.052

Liu, S., Wang, Z., \& Li, X. (2014). Long-term properties of concrete containing ground granulated blast furnace slag and steel slag. Magazine of Concrete Research, 66(21), 1095-1103. doi:10.1680/ macr.14.00074

Mathew, G., \& Paul, M. (2014). Influence of fly Ash and GGBFS in laterized concrete exposed to elevated temperatures. Journal of Materials in Civil Engineering, 26(3), 411-419. doi:10.1061/ (ASCE)MT.1943-5533.0000830

Mohamed, O., \& Al Hawat, W. (2018). Durability and Strength of Sustainable Self-Consolidating Concrete Containing Fly Ash. IOP Conference Series: Materials Science and Engineering, 324, p. 012041. Emiratos Arabes Unidos. doi: 10.1088/1757899X/324/1/012041

Mohamed, O., \& Najm, O. (2017). Compressive strength and stability of sustainable self-consolidating concrete containing fly ash, silica fume, and GGBS. Frontiers of Structural and Civil Engineering, 11(4), 1-6. doi:10.1007/s11709-016-0350-1

Nagrockiene, D., Rutkauskas, A., Pundiene, I., \& Girniene, I. (2019). The Effect of Silica Fume Addition on the Resistance of Concrete to Alkali Silica Reaction. IOP Conference Series: Materials Science and Engineering, 660. Lituania. doi:10.1088/1757899X/660/1/012031

Nuaklong, P., Wongsa, A., Sata, V., Boonserm, K., Sanjayan, J., \& Chindaprasirt, P. (2019). Properties of high-calcium and lowcalcium fly ash combination geopolymer mortar containing recycled aggregate. Heliyon, 5(9), 02513. doi:10.1016 / j.heliyon.2019.e02513

Pereira, A., da Silva, C., de Araújo Queiroz, D., de Moraes, M., Melges, J., Tashima, M., \& Akasaki, J. (2015). Estudo das propriedades mecânicas do concreto com adição de cinza de casca de arroz. Revista Materia, 20(1), 227-238. doi:10.1590/ S1517-707620150001.0023

Reddy, D., \& Ramaswamy, A. (2017). Influence of mineral admixtures and aggregates on properties of different concretes under high temperature conditions I: Experimental study. Journal of Building Engineering, 14, 103-114. doi:10.1016/j. jobe.2017.09.013
Rodrigues, M., \& Beraldo, A. (2010). Caracterização física e mecânica de argamassas à base de cimento Portland e cinza de casca de arroz residual. Engenharia Agricola, 30(2), 193-204. doi:10.1590/s0100-69162010000200001

Saba , A., \& Zeyad, A. (2018). Influence of pulverized fly ash on the properties of self-compacting fiber reinforced concrete. Scientific Journal of King Faisal University, 19(2), 55-68.

Sanjay , R., Vijayan, D., Mubarak , P., Subinjith, N., \& Santhosh, S. (2020). Effect of silica fume on strength of glass fiber incorporated concrete. AIP Conference Proceedings, 2271, p. 30020. doi:10.1063/5.0024775

Santhosh, S., \& Arivalagan, S. (2018). Investigation of m-sand concrete mix with mineral admixtures as partial replacement of cement. International Journal of Civil Engineering and Technology, 9(12), 712-719.

Sathawane, S., Vairagade, V., \& Kene, K. (2013). Combine Effect of Rice Husk Ash and Fly Ash on Concrete by 30\% Cement Replacement. Procedia Engineering, 51, 35-44. doi:10.1016/ j.proeng.2013.01.009

Shi, Y., Li, X., Li, Y., Peng, Z., \& Li, J. (2020). Effect of Tuff Powder Mineral Admixture on the Macro-Performance and Micropore Structure of Cement-Based Materials. Frontiers in Materials, 7, 595997. doi:10.3389 / fmats.2020.595997

Siad, H., Mesbah, H., Mouli, M., Escadeillas, G., \& Khelafi, H. (2014). Influence of Mineral Admixtures on the Permeation Properties of Self-Compacting Concrete at Different Ages. Arabian Journal for Science and Engineering, 39(5), 3641-3649. doi:10.1007/s13369-014-1055-1

Singh, S., Ransinchung, G., \& Kumar, P. (2017). Effect of mineral admixtures on fresh, mechanical and durability properties of RAP inclusive concrete. Construction and Building Materials, 156(15), 19-27. doi:10.1016/j.conbuildmat.2017.08.144

Soares dos Anjos, M. A., Farias, E. C., Ferreira, R., \& Pederneiras, C. M. (2020). Analysis Of Mechanical Properties And Durability Of Selfcompacting. In book: NEW TRENDS IN GREEN CONSTRUCTION.

Sukontasukkul, P., Chindaprasirt, P., Pongsopha, P., Phoo-Ngernkham, T., Tangchirapat, W., \& Banthia, N. (2020). Effect of fly ash/ silica fume ratio and curing condition on mechanical properties of fiber-reinforced geopolymer. Journal of Sustainable CementBased Materials, 9(4), 1-15. doi:10.1080/21650373.2019.17099 99

Temiz, H., \& Kantarci, F. (2014). Investigation of durability of CEM II B-M mortars and concrete with limestone powder, calcite powder and fly ash. Construction and Building Materials, 68, 517-524. doi:10.1016/j.conbuildmat.2014.06.078 
Toshiki , A., \& Takashi , F. (2021). Improvement of Concrete Properties using Granulated Blast Furnace Slag Sand. Journal of Advanced Concrete Technology, 19(2), 118-132. doi:10.3151/ jact.19.118

Wang, X.-Y., \& Park, K.-B. (2015). Analysis of compressive strength development of concrete containing high volume fly ash. Construction and Building Materials, 98, 810-819. doi:10.1016/j. conbuildmat.2015.08.099

Zhu, Y., Zhang, Z., Yang, Y., \& Yao, Y. (2014). Measurement and correlation of ductility and compressive strength for engineered cementitious composites (ECC) produced by binary and ternary systems of binder materials: Fly ash, slag, silica fume and cement. Construction and Building Materials, 68, 192-198. doi:10.1016/j.conbuildmat.2014.06.080 